\title{
Content coverage in Nature Clinical Practice Urology: a balancing act
}

\author{
Alexandra M Hay and Sandra A Ford
}

One of the challenges every journal editor has is achieving an even coverage of different subspecialties across issues. Having balanced issues is important to Nature Clinical Practice Urology in order to be fully representative of the discussions and developments across urology and related specialties. We understand that many of our readers are specialists, and we aim to provide articles that are of a broad interest as well as those suited to a more specialist audience.

To achieve the broadest coverage of urology subspecialties the editorial team regularly scan 65 international journals in order to identify research papers of relevance for the Research Highlights and Practice Points sections of the journal. Achieving balanced coverage can sometimes be difficult, especially when the proportion of research studies published in the urology field is heavily weighted towards oncology. This poses us with a dilemma when selecting which research papers to cover, raising two important questions: should we sacrifice a balance of topics to ensure we include more high-quality, ground-breaking oncology papers, or should we cover potentially weaker studies in order to achieve a coverage that is as representative of as many subspecialties as possible?

There is no simple answer, and we will continue to cover as many subspecialties as possible with our Practice Point commentaries from authors worldwide, balancing paper selection with our requirements for covering the highest-quality research in a timely manner. In order for us to achieve this, you may find that some of our forthcoming issues have a higher number of oncology-related Practice Point articles than previously seen.

In this issue we are able to present a good balance of topics, and we hope you enjoy the

\section{Achieving \\ balanced \\ coverage can \\ sometimes \\ be difficult, especially \\ when the \\ proportion \\ of research \\ studies \\ published in \\ the urology \\ field is heavily \\ weighted \\ towards \\ oncology.}

AM Hay is Editor and

$S A$ Ford is Associate

Editor of Nature

Clinical Practice

Urology, Nature

Publishing Group,

London, UK.

\section{Competing interests}

The authors declared

they have no competing

interests.

www.nature.com/clinicalpractice doi:10.1038/ncpuro0400 wide range of areas covered herein. The subject of nephrectomy is comprehensively covered, with a Viewpoint, Practice Point and Review. Paul Russo weighs in on the debate regarding open versus laparoscopic surgery for renal tumors, arguing that the benefits of open partial nephrectomy are being passed over in favor of more radical laparoscopic surgery. Howard Winfield's Practice Point assesses the results of a study examining the retroperitoneoscopic approach to living donor nephrectomies. The topic is rounded off with a review by Nasser Albqami and Günter Janetschek discussing the indications and contraindications for laparascopic nephrectomy in the treatment of renal cell carcinoma.

Female urology is also covered, with a commentary by authors from the Virginia Mason Medical Center on the recent finding that weight loss - through diet and exercise alonecould serve as an effective treatment for urinary incontinence in overweight and obese women. The importance of this finding is particularly pertinent as the obesity epidemic in western countries continues. A review by Gary Lemack examines the assessment and diagnosis of bladder outlet obstruction in women, a condition that is difficult to diagnose but that is becoming widely recognized in women undergoing urodynamic investigations for lower urinary tract symptoms.

We hope you enjoy reading these commentaries and reviews, and the other excellent articles included in this journal issue. We can assure you that the editorial team and our international Advisory Board of experts will continue to cover a broad range of topics, ensuring that we reach as many of our readers' needs as possible. 